\title{
Luminosity function for galaxy clusters
}

\author{
K. Bajan ${ }^{1}$, M. Biernacka ${ }^{2}$, P. Flin ${ }^{2}$, W. Godłowski ${ }^{3}$, E. Panko ${ }^{4}$ and \\ J. Popiela ${ }^{3}$ \\ ${ }^{1}$ Mt. Suhora Observatory, Cracow Pedagogical University, Krakow, Poland, \\ email: kbajan@up.krakow.pl \\ ${ }^{2}$ Institute of Physics, Jan Kochanowski University, Kielce, Poland \\ ${ }^{3}$ Institute of Physics, Opole University, Opole, Poland \\ ${ }^{4}$ Kalinenkov Observatory, Nikolaev National University, Nikolaev, Ukraine
}

\begin{abstract}
We constructed and studied the luminosity function of 6188 galaxy clusters. This was performed by counting brightness of galaxies belonging to clusters in the PF catalogue, taking galaxy data from MRSS. Our result shows that the investigated structures are characterized by a luminosity function different from that of optical galaxies and radiogalaxies (Machalski \& Godłowski 2000). The implications of this result for theories of galaxy formation are briefly discussed.
\end{abstract}

\section{Introduction}

For galaxies, the luminosity function is usually described by the Schechter function (Schechter 1976). This function presents the space density of objects as function of their luminosities. The first parameter has units of number density and provides the normalization. The galaxy luminosity function may have different parameters for different populations and environments; it is not a universal function. One measurement based on field galaxies is (Machalski \& Godłowski 2000):

$$
\alpha=-1.25, \phi^{*}=1.2 \times 10^{-3} h^{3} M p c^{-3} .
$$

It is often convenient to rewrite the Schechter luminosity function in terms of magnitude, rather than luminosities. In this case the function becomes:

$$
n(M) d M=0.4 \ln 10 \phi^{*}\left[10^{-0.4\left(M-M^{*}\right)}\right]^{\alpha+1} \exp \left[-10^{-0.4\left(M-M^{*}\right)}\right] d M
$$

\section{Observational data}

The catalogue of 6188 galaxy clusters by Panko \& Flin (2006) (hereafter PF) served as the observational basis for our studies. It was prepared by applying Voronoi tessellation (Ramella et al. 1999, 2001) to the Muenster Red Sky Survey (MRSS, Ungruhe et al. 2003). The PF catalogue is statistically complete up to $r_{F}=18.3^{m}$. Each cluster contains at least 10 galaxies in the brightness range $m_{3}, m_{3}+3^{m}$, where $m_{3}$ is the magnitude of the third brightest galaxy in the cluster. The distances to the clusters were determined using the relation between the tenth brightest galaxy $m_{10}$ and redshift (Panko et al. 2009).

\section{Results}

In the case where $m_{3}$ is good indicator of distance, Fig. 1a,b represents the luminosity function. Fig.1c,d presents the distribution of absolute magnitudes of $m_{3}$. The dispersion 

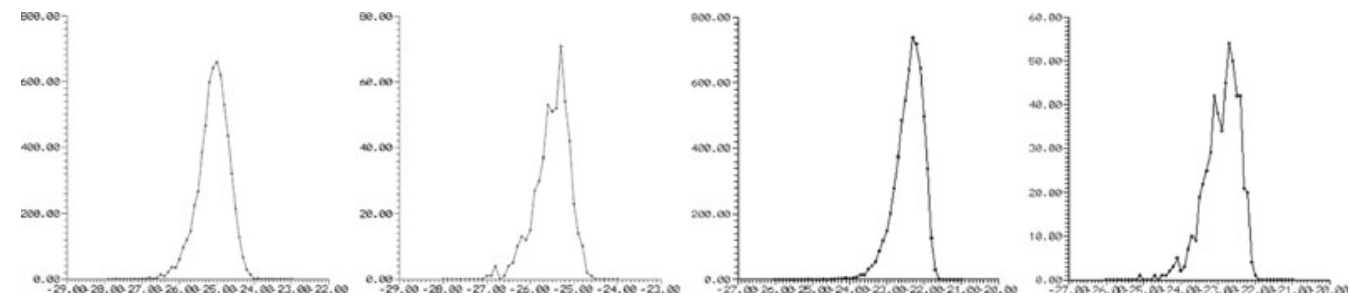

Figure 1. Histograms: number of clusters versus absolute magnitude (First left (1a) - for all clusters; second left $(1 \mathrm{~b})$ - for the statistically complete sample, $m_{3}<15.3$ ). Histograms of the absolute magnitude of the $m_{3}$ galaxy in each cluster for all clusters and for the statistically complete sample - $1 \mathrm{c}, \mathrm{d}$.

is clearly observed. In order to correct for this effect we applied Condon's (1989) method, which consists of weighting each cluster by a weight reversely proportional to the cluster volume $V_{\max }$, where $V_{\max }$ is connected with the cluster redshift.

The Condon-method corrected histograms were quite similar to those presented in Fig. 1a,b. The calculated values of the parameters are: $M^{*}=-23.1, \alpha=4.26$. These values are drastically different from the corresponding values for galaxies see eq. (1.1). On the other hand they are quite close to the values obtained without taking into account the distribution of $m_{3}$. These values were also counted using a different approach for the Schechter luminosity function determination, namely that of Efstathiou et al. (1988) and Lin \& Kirshner (1996), where the maximum likelihood method is applied. Their values was quite similar to those given above.

\section{Conclusions}

- The third-brightest galaxies in clusters have different absolute magnitudes.

- The cluster luminosity function is significantly different than that obtained for both optical and radiogalaxies (Machalski \& Godłowski 2000).

- The shape of the cluster luminosity function, as obtained through this method, is a Gaussian, not a Schechter function.

- The application of Condon method does not change drastically the obtained result.

- A possible explanation of the difference in cluster and galaxy luminosity functions are the different scenarios leading to the formation of these structures (galaxies and clusters).

\section{References}

Condon, J. J. 1989, ApJ 338, 13

Efstathiou, G., Ellis, R. S., \& Peterson, B. A. 1988, MNRAS 232, 431

Lin, H. \& Kirshner, R. P. 1996, ApJ 464, 60

Machalski, J. \& Godłowski, W. 2000, AA 360, 463

Panko, E. \& Flin, P. 2006, Journal Astron. Data, 12, 1

Panko, E., Juszczyk, T., Biernacka, M., \& Flin, P. 2009, ApJ 700, 168

Ramella, M., Nonino, M., Boschin, W., \& Fadda, D. 1999, in: ASP Conf. Ser. 176, Observational Cosmology: The Development of Galaxy Systems, ed. G. Giuricin,M. Mezzetti, \& P. Salucci (San Francisco: ASP), s.108

Ramella, M., Boschin, W., Fadda, D., \& Nonino, M. 2001, A\& A, 368, 776

Schechter, P. 1976, ApJ, 203, 297

Ungruhe, R., Seitter, W. C., \& Durbeck, H. W. 2003, Journal Astronomical Data 9, 1 